\section{- OPEN ACCESS}

\title{
Occupational exposure of healthcare and research staff to static magnetic stray fields from 1.5-7 Tesla MRI scanners is associated with reporting of transient symptoms
}

\author{
Kristel Schaap, ${ }^{1}$ Yvette Christopher-de Vries, ${ }^{1}$ Catherine K Mason, ${ }^{1}$ Frank de Vocht, ${ }^{2}$ \\ Lützen Portengen, ${ }^{1}$ Hans Kromhout ${ }^{1}$
}

- Additional material is published online only. To view please visit the journal online (http://dx.doi.org/10.1136/ oemed-2013-101890).

1 Division of Environmental Epidemiology, Institute for Risk Assessment Sciences, Utrecht University, Utrecht,

The Netherlands

${ }^{2}$ Centre for Occupational and Environmental Health, Centre of Epidemiology, Institute of Population Health, Manchester Academic Health Sciences Centre, The University of Manchester, Manchester, UK

\section{Correspondence to} Professor Hans Kromhout, Institute for Risk Assessment Sciences, Utrecht University, PO Box 80178, Utrecht 3508 TD, The Netherlands; h.kromhout@uu.nl

Received 27 September 2013 Revised 26 February 2014 Accepted 16 March 2014 Published Online First 8 April 2014

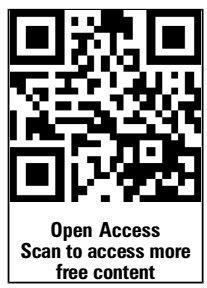

CrossMark

\begin{tabular}{l}
\hline To cite: Schaap K, \\
Christopher-de Vries Y, \\
Mason CK, et al. Occup \\
Environ Med 2014;71: \\
423-429. \\
\hline
\end{tabular}

\section{ABSTRACT}

Objectives Limited data is available about incidence of acute transient symptoms associated with occupational exposure to static magnetic stray fields from MRI scanners. We aimed to assess the incidence of these symptoms among healthcare and research staff working with MRI scanners, and their association with static magnetic field exposure.

Methods We performed an observational study among 361 employees of 14 clinical and research MRI facilities in The Netherlands. Each participant completed a diary during one or more work shifts inside and/or outside the MRI facility, reporting work activities and symptoms (from a list of potentially MRI-related symptoms, complemented with unrelated symptoms) experienced during a working day. We analysed 633 diaries. Exposure categories were defined by strength and type of MRI scanner, using non-MRI shifts as the reference category for statistical analysis. Non-MRI shifts originated from MRI staff who also participated on MRI days, as well as CT radiographers who never worked with MRI. Results Varying per exposure category, symptoms were reported during $16-39 \%$ of the MRI work shifts. We observed a positive association between scanner strength and reported symptoms among healthcare and research staff working with closed-bore MRI scanners of 1.5 Tesla ( $\mathrm{T}$ ) and higher (1.5 T OR=1.88; 3.0 T OR=2.14; 7.0 T $\mathrm{OR}=4.17)$. This finding was mainly driven by reporting of vertigo and metallic taste.

Conclusions The results suggest an exposure-response association between exposure to strong static magnetic fields (and associated motion-induced time-varying magnetic fields) and reporting of transient symptoms on the same day of exposure.

Trial registration number $11-032 / \mathrm{C}$

\section{INTRODUCTION}

Individuals who work in close proximity to a MRI scanner are exposed to electromagnetic fields (EMF) from the MRI scanner. A major group of people regularly exposed to MRI-related EMF are healthcare and research staff. MRI staff are mainly exposed to the static magnetic field (SMF) produced by the magnet of the MRI scanner and, additionally, experience low-frequency time-varying magnetic fields (TvMF) from movement through the static magnetic stray field around the scanner.

\section{What this paper adds}

- Limited data is available about the incidence of acute short-lasting symptoms among clinical and research staff working with MRI scanners.

- This study among 361 workers indicates an increased incidence of specific transient symptoms among MRI staff working with closed-bore MRI scanners of 1.5-7 Tesla, and suggests a positive exposure-response association with exposure to static magnetic fields and/or motion-induced time-varying magnetic fields.

- The recently accepted European Union Physical Agents Directive (Directive 2013/35/EU) exempts MRI workers from EMF exposure limits. However, during $6 \%$ of the MRI shifts in this study workers experienced vertigo, which constitutes a potential safety hazard for both worker and patient. Additionally, several workers reported that symptoms affected their ability to work. With MRI system magnets getting increasingly stronger, the number of workers experiencing MRI-related symptoms is expected to increase. The results of this study suggest that protocols to ensure a safe and healthy work environment are clearly needed.

Additional exposure to switched gradient fields (SGF), which are applied for spatial encoding of the image, is possible in some cases when staying close to the scanner during image acquisition. ${ }^{1}$ SGFs are also TvMFs, but unlike exposures resulting from motion in an MRI scanner's static magnetic stray field, SGFs are fields with well-defined frequency spectra $(\mathrm{kHz}$ range) that can vary with the MRI's imaging protocol. ${ }^{2}$ Neural responses and physiological effects of exposure to TvMFs strongly depend on the spatial and temporal characteristics of the magnetic field stimulus. ${ }^{3}$ As a result, even though SGFs and motion-induced TvMF are both TvMFs, different effects on the body can be expected from exposure to these fields. ${ }^{2}{ }^{4}$ Further use of the term TvMF in this paper will refer to motion-induced TvMF. 
Exposure to SGFs can cause peripheral nerve stimulation at specific pulse sequences, which can be experienced as a tingling sensation or muscle contractions. ${ }^{245}$ Various publications have described subjective symptoms in relation to SMF exposure, reported by people who either work near an MRI scanner ${ }^{6-10}$ or who are exposed to MRI-related EMFs as scanned patient or healthy volunteer. ${ }^{71-16}$ Evidence originates from experimental as well as observational studies and includes general symptoms, such as headache and concentration problems, as well as specific 'sensory' symptoms such as vertigo, balance problems, nausea, metallic taste and seeing light flashes (magnetophosphenes). Current literature suggests that these symptoms have an acute and transient nature, ${ }^{10}{ }^{16-18}$ and many of these arise when people move through the spatial gradients in the static magnetic stray field outside the MRI scanner, resulting in exposure to lowfrequency TvMFs. ${ }^{14} 1718$ In only a few studies were symptoms assessed in a (semi-)objective way, either by blinding subjects to the exposure condition, or by making inquiries about symptoms during the work day in general as opposed to specifically relating it to MRI or SMF exposure. ${ }^{8} 151719$ Furthermore, in only a few studies was symptom occurrence related to different exposure conditions using statistical tests. ${ }^{8} 9141520$ Consequently, current literature has been inconclusive about associations of specific symptoms with MRI-related electromagnetic field exposure, especially in relation to exposure to strong SMFs.

Incidence of symptoms among clinical and research MRI staff has not yet been extensively studied. In a small survey of 59 Swedish MRI nurses, 15 percent had experienced at least one symptom thought to originate from their work near the MRI scanner, and symptom incidence increased with scanner field strength from $13 \%$ at 1 Tesla to $57 \%$ at 3 Tesla. ${ }^{10}$ Two other studies investigated the occurrence of acute symptoms among a smaller sample ( $n=15$ and 20 ) of MRI staff working with clinical or research scanners up to 7 Tesla, and found that between $15 \%$ and $75 \%$ of people working with scanners between 1.5 and 7 Tesla, respectively, experienced metallic taste and/or vertigo. $^{6} 7$

We designed an observational study among 14 clinical and research MRI facilities, aiming to gain further insight into the incidence and duration of acute symptoms experienced by MRI staff, and to assess how the incidence of these symptoms is associated with level of exposure to SMFs.

\section{METHODS}

\section{Study sample and data collection}

The study was performed in four general hospitals, four academic hospitals, one academic children's hospital, one human neuroscientific research institute and four animal MRI research units in The Netherlands. These 14 clinical and research MRI facilities were visited for 1 or 2 weeks between March 2011 and February 2012. This study was part of a larger study that was aimed at assessment of SMF exposure determinants. Towards that purpose, facilities had been selected in order to represent a broad selection of MRI applications, job titles, scanners and other potential exposure determinants. As a result, especially academic hospitals and research MRI facilities were oversampled in comparison to the total population of 152 MRI departments in The Netherlands. A complete overview of the number of MRI departments, MRI scanners and exposed workers in The Netherlands was described by Schaap et al. ${ }^{21}$ All employees who worked at the MRI facility during the week(s) of the visit were asked to complete a work-related diary for at least one MRI work shift. Employees included MRI radiographers, but also other clinical staff, researchers and technical support staff. Eligible employees were given the opportunity to refuse participation when approached. Where possible, each participant also completed a diary on a working day when he or she did not work with or around an MRI-system, to serve as a within-subject control condition. Because the number of participants who completed a diary on an SMF unexposed day was low, an additional SMF unexposed group was included consisting of CT radiographers who never worked with MRI systems. These CT radiographers were asked to complete a diary during one working day at the CT facility.

The questions in the diaries referred to the full work shift of the participant. Exposure-related questions about the participant's working day included questions about their presence in the MRI scanner room, the type of scanner worked with, and presence in a scanner room during image acquisition.

Additionally, participants were asked to report which out of 21 symptoms they had experienced before the start of their shift (as a baseline) and during their work shift. Participants also reported the duration of each symptom, whether these symptoms affected their work performance, and what they thought was causing the symptoms. Symptoms explained by previous injury or a pre-existing condition (eg, chronic tinnitus) were excluded from analysis based on the latter question. Information on potential confounding or effect-modifying covariates included gender, age, the use of cleaning agents and solvents during the working day, alcohol consumption during the previous $24 \mathrm{~h}$ and the subjects' perception of their workload (light, moderate or heavy). The exact questions in the diary can be found in the online supplementary eAppendix 1.

To aid recall of the tasks performed and symptoms experienced during the shift, participants who spent their complete work shift at an MRI or CT facility (MRI and CT radiographers and some anaesthesiologists) filled in their diary at two time points per shift. These forms were combined to derive a fullshift report. Because of the more incidental and sometimes taskbased nature of their work at the MRI facility, other employees, such as medical doctors, anaesthesiologists and researchers filled in only one form for their full shift.

Exposure to SMFs-which coincides with exposure to TvMF due to movement through the spatial gradients of the SMFwas defined as presence inside the MRI scanner room for any period of time during the shift. Based on this definition, participants were assigned to an SMF exposed group or to an SMF unexposed group. Members of the SMF exposed group were also assigned to one of five scanner categories based on a combination of the field strength and model of the scanner with which they worked during their shift. On the occasions that they worked with multiple scanners of the same model (ie, closed-bore scanners), the scanner with the highest field strength was used for exposure classification. When participants worked with multiple scanners of different models, they were assigned to multiple scanner categories, since scanner strengths of different models are not comparable with respect to SMF exposure. Potential exposure to SGFs, which can result from standing close to an MRI scanner while it is performing a scan, was also assessed and was defined as presence in an MRI scanner room during image acquisition.

Twenty-one symptoms were included in the analysis: $13 \mathrm{SMF}$ target symptoms, 2 SGF target symptoms and 6 unrelated symptoms that were assumed to have no relation with SMF or SGF exposure (table 1). The $13 \mathrm{SMF}$ target symptoms had been reported previously in association with exposure to MRI-related SMF and/or TvMF. ${ }^{7-12}{ }^{14-17}$ A subset of 5 of these 13 symptoms (vertigo, metallic taste, nausea, magnetophosphenes and head 
Table 1 Symptoms and symptom categories included in the study

\begin{tabular}{|c|c|c|}
\hline Symptom as described in diary & Shortened name & Category \\
\hline Sensation of dizziness or vertigo & Vertigo & SMF target-core \\
\hline Nausea & Nausea & SMF target-core \\
\hline Tinnitus/sensation of head ringing & Head ringing & SMF target-core \\
\hline Seeing light spots or light flashes & Magnetophosphenes & SMF target-core \\
\hline Metallic taste & Metallic taste & SMF target-core \\
\hline Headache & Headache & SMF target \\
\hline Tiredness or sleepiness & Tiredness & SMF target \\
\hline Concentration problems & Concentration problems & SMF target \\
\hline Vomiting & Vomiting & SMF target \\
\hline Feeling of instability when standing, walking or moving & Instability & SMF target \\
\hline Feeling lightheaded or weightless & Lightheadedness & SMF target \\
\hline Blurred or double vision & Blurred vision & SMF target \\
\hline A strange smell sensation & Strange smell & SMF target \\
\hline Tingling sensation in the body & Tingling & SGF target \\
\hline Involuntary muscle contractions & Muscle contractions & SGF target \\
\hline Seeing black spots or having a temporary loss of vision & Black spots & A priori unrelated \\
\hline Itchy, watery or red eyes & Irritated eyes & A priori unrelated \\
\hline Sensation of glowing, burning or irritated skin & Irritated skin & A priori unrelated \\
\hline Suddenly feeling warm or hot, hot flashes & Hot flashes & A priori unrelated \\
\hline Earache & Earache & A priori unrelated \\
\hline Palpitation & Palpitation & A priori unrelated \\
\hline
\end{tabular}

ringing), were identified as being of greater interest due to stronger evidence from prior research ${ }^{8}{ }^{15}$ for their association with SMF and TvMF exposure. These symptoms were classified as SMF target 'core' symptoms. Two SGF target symptoms related to peripheral nerve stimulation were included for the clinical and research staff that might sometimes be exposed to SGFs: A tingling sensation in the body and involuntary muscle contractions. ${ }^{5}$ Six generally occurring symptoms (seeing black spots, irritated eyes, irritated skin, hot flashes, earache and palpitations) categorised as 'a priori unrelated symptoms' in table 1, were a priori assumed to be unrelated to SMF and SGF exposure, and were included to assess potential over-reporting of symptoms in association with SMF exposure. Binary category scores (ie, reporting any of the symptoms within a symptom category) were calculated for each of the four symptom categories.

All participants gave informed consent for participation in the study and were asked to fill in a general questionnaire to obtain information about gender, age and job title (see online supplementary eAppendix 2). This study was approved by the Medical Research Ethics Committee of the Utrecht University Medical Center.

\section{Statistical analysis}

Symptom incidence and associations between symptom incidence and exposure were analysed at work-shift level. Because initial analyses showed strong clustering of symptom reporting within subjects (ie, high between-subjects heterogeneity in reporting of symptoms), we used a finite mixture (FM) model to test associations between scanner categories and symptoms, with subjects incorporated as random effects in the model. FM models are similar to generalised linear mixed models (GLMM), but with random effects assumed to be from a discrete distribution (instead of a normal distribution as in GLMMs). Conceptually, these models may be derived from the assumption that each subject belongs to one of several (latent) classes, and that the multiple responses for a subject are generated according to a class-specific model. We used the FlexMix package (V.2.3$8)^{22-24}$ in R (V.2.15.2, R Foundation for Statistical Computing, Vienna, Austria) to estimate the number of (latent) classes and the parameters of the concomitant (predicting class membership) and class-specific (predicting symptom reporting) models. Model selection was based on the Akaike Information Criterion (AIC). ${ }^{25}$ Variables that significantly affected the model fit were included in the model. A 2-class model that included gender and age as predictors for class membership and workload, use of solvents and alcohol consumption as confounders in the symptom prediction model best fitted the data. One of the classes had a very low probability of reporting symptoms (henceforth called the 'non-reporting' class), while reporting in the other ('symptom-reporting') class was low during SMF unexposed shifts but significantly higher during exposed shifts.

\section{RESULTS}

Over $95 \%$ of the employees who were approached volunteered to participate in the study. This resulted in a total of 361 participants who completed 681 diaries. Diaries that were not representative for the duration of the full shift $(n=3)$, diaries completed by CT radiographers who had entered an MRI scanner room during their shift $(n=3)$, and diaries that contained incomplete data on exposure status (SMF exposed, SMF unexposed) or symptoms $(n=42)$ were excluded from analysis. This resulted in a final number of 633 observations from 331 subjects. Participants completed 1-6 diaries, with 51\% completing only one diary. Mean age of the study participants was 35.7 years $(\mathrm{SD}=10.3)$, with $57 \%$ of the participants being women. Further description of the study population and work shift characteristics can be found in table 2. Scanner field strength ranged from 0.5 Tesla to 11.7 Tesla, with 1.5 Tesla closed-bore clinical scanners being most frequently reported (38\% of shifts).

When stratified by scanner category, SMF target symptoms were reported during 16-39\% of the shifts (overall 26\%) (table 3). 
Table 2 Description of the collected data

\begin{tabular}{|c|c|c|c|c|}
\hline & \multicolumn{2}{|c|}{$\begin{array}{l}\mathrm{n}_{\text {subj }}(\%) \\
\text { Total=331 }\end{array}$} & \multicolumn{2}{|c|}{$\begin{array}{l}\mathrm{n}_{\text {obs }}(\%) \\
\text { Total }=633\end{array}$} \\
\hline \multicolumn{5}{|l|}{ Job title } \\
\hline Radiographer, radiography student or intern* & 197 & $(60)$ & 418 & (66) \\
\hline Scientific staff or research student & 101 & (31) & 172 & (27) \\
\hline Clinical staff & 19 & (6) & 21 & (3) \\
\hline Technical staff & 10 & (3) & 17 & (3) \\
\hline Unknown & 4 & (1) & 5 & (1) \\
\hline \multicolumn{5}{|l|}{ Gender } \\
\hline Female & 188 & $(57)$ & 354 & (56) \\
\hline Male & 139 & $(42)$ & 274 & (43) \\
\hline Unknown & 4 & (1) & 5 & (1) \\
\hline \multicolumn{5}{|l|}{ Scanner category $\dagger$} \\
\hline Unexposed & 129 & (39) & 134 & (20) \\
\hline $1.5 \mathrm{~T}$ closed bore $\ddagger$ & 142 & (43) & 259 & (38) \\
\hline 3.0 T closed bore§ & 89 & $(27)$ & 131 & (19) \\
\hline 7.0 T closed bore & 25 & (8) & 31 & (5) \\
\hline Various scanner types up to $1.5 \mathrm{~T}^{* *}$ & 34 & $(10)$ & 49 & (7) \\
\hline Small-bore animal scanners $>4.7 \mathrm{~T} \dagger \dagger$ & 34 & $(10)$ & 57 & (8) \\
\hline Unknown & 13 & (4) & 14 & (2) \\
\hline \multicolumn{5}{|l|}{ Reported workload levelt } \\
\hline Low & 135 & $(41)$ & 180 & (28) \\
\hline Medium & 226 & $(68)$ & 366 & (58) \\
\hline High & 72 & $(22)$ & 86 & (14) \\
\hline Unknown & 1 & (0) & 1 & (0) \\
\hline \multicolumn{5}{|l|}{ Used disinfectants or solvents during workt } \\
\hline Yes & 235 & $(71)$ & 417 & (66) \\
\hline No & 141 & (43) & 210 & (33) \\
\hline Unknown & 5 & (2) & 6 & (1) \\
\hline \multicolumn{5}{|l|}{ Consumed alcohol during $24 \mathrm{~h}$ prior to shift } \\
\hline Yes & 124 & (38) & 165 & (26) \\
\hline No & 257 & (78) & 438 & (69) \\
\hline Unknown & 22 & (7) & 30 & (5) \\
\hline \multicolumn{5}{|c|}{$\begin{array}{l}\text { *This group includes } 68 \text { observations obtained from } 67 \text { CT radiographers and CT } \\
\text { radiography interns. The remaining observations are from MRI radiographers, students } \\
\text { and interns. } \\
\text { tDue to multiple observations per subject, a subject can appear in more than one } \\
\text { category. Therefore, the sum might exceed } 100 \% \text {. This also applies to the number of } \\
\text { shifts per scanner category, since within a shift a subject could have worked with } \\
\text { more than one type of scanner. } \\
\text { fIncludes all large-bore cylindrical systems of } 1.5 \text { Tesla. } \\
\text { SIncludes all large-bore cylindrical systems of } 3.0 \text { Tesla. } \\
\text { IIncludes all large-bore cylindrical systems of } 7.0 \text { Tesla. } \\
\text { **Includes extremity scanners, upright scanners and open (ie, transversal field) } \\
\text { scanners up to } 1.5 \text { Tesla and large-bore cylindrical systems below } 1.5 \text { Tesla. } \\
\text { t†Includes small-bore cylindrical animal scanners with field strengths between } 4.7 \\
\text { and } 11.7 \text { Tesla. } \\
\mathrm{N}_{\text {subj, number of subjects; } \mathrm{N}_{\text {obs, }} \text { number of observations; T, Tesla. }}\end{array}$} \\
\hline
\end{tabular}

During only $10 \%$ of all shifts, subjects reported more than 1 SMF target symptom. Two target symptoms were not reported by the study sample: Vomiting and involuntary muscle contractions. Other target symptoms were reported during $0.2-12 \%$ of the shifts.

SMF core symptoms were reported during $6 \%$ of the shifts. In only $2 \%$ of all shifts, subjects reported more than $1 \mathrm{SMF}$ core symptom. The most frequently reported core symptom was vertigo ( $4 \%$ of all shifts).

Potential exposure to SGFs occurred during 13\% of the shifts. Only two participants reported an SGF target symptom (a tingling sensation). One of these participants had reported to have been present inside a scanner room during image acquisition and experienced a tingling sensation while standing next to the bore entrance for several minutes, providing a tactile stimulus to a volunteer undergoing a functional MRI (fMRI) scan.

A full overview of the overall reporting frequencies of all symptoms, including stratification by exposure condition, can be found in the online supplementary material eTable S1.

Table 4 presents the results of the symptom prediction models in the 'symptom-reporting' class comparing SMF exposed and unexposed subjects per scanner category, adjusted for gender, age, workload, use of solvents and alcohol ingestion. ORs from the adjusted models were similar to those from unadjusted or partly adjusted models (data not shown). SMF target symptoms were more frequently reported by SMF-exposed subjects working with 1.5 Tesla, 3 Tesla and 7 Tesla closed-bore scanners. ORs for the closed-bore scanner categories increased with increasing scanner field strengths (1.5 Tesla closed bore OR (95\% CI) 1.88 (1.07 to 3.31 ); 3.0 Tesla closed bore OR (95\% CI) 2.14 (1.13 to 4.03); 7.0 Tesla closed bore OR (95\% CI) $4.17(1.30$ to 13.35$))$. Also when only the subgroup of SMF core symptoms was considered, increased reporting of symptoms among subjects working with 3 Tesla and 7 Tesla closed-bore scanners was observed. Moreover, when compared to SMF target symptoms, ORs for SMF core symptoms were larger for all closed-bore scanners and showed an even stronger exposure-depending increase (1.5 Tesla closed bore OR $(95 \%$ CI) 2.41 (0.72 to 8.13); 3.0 Tesla closed bore OR $(95 \% \mathrm{CI})$ 4.92 (1.21 to 19.97); 7.0 Tesla closed bore OR (95\% CI) 66.82 (6.39 to 698.97$))$.

There was no suggestion of increased symptom reporting among subjects working with low-field scanners of various types up to 1.5 Tesla (including closed-bore, open, upright and extremity scanners) or small-bore animal scanners (4.711.7 Tesla). Subjects working with small-bore animal scanners had the lowest rate of reporting SMF target symptoms (16\% of shifts), and none of the SMF core symptoms were reported by these subjects. A priori unrelated symptoms were reported at similar frequencies as the unexposed group (table 4).

Due to low prevalence of individual symptoms, and in order to avoid testing a large number of associations (ie, multiple comparisons), which would greatly reduce statistical power, associations were not statistically tested per individual symptom. However, visual inspection of crude incidence of individual symptoms among the various scanner categories suggests that the trend of increased symptom reporting with increasing closed-bore scanner field strength was predominantly driven by the core symptoms of vertigo, metallic taste and nausea with nausea showing the least pronounced trend. These symptoms were reported during 5.6\%, $1.8 \%$ and $1.2 \%$ of the MRI shifts, respectively, and were absent during non-MRI shifts. Reported durations of these symptoms ranged between less than $1 \mathrm{~min}$ and 5-15 min. A full overview of crude incidence of individual symptoms per scanner category and most reported duration per symptom can be found in the online supplementary material (eTables S2 and S3).

Participants were asked whether and how their work practice had been affected by the symptoms they experienced. Ten (10\%) out of 103 SMF-exposed participants who reported any of the SMF or SGF target symptoms indicated that their work practice had been affected by one or more of the symptoms they had experienced. These subjects had worked with 1.5-7 Tesla closed-bore scanners. Most subjects $(n=7)$ stated that the symptoms affected their concentration, work pace or efficiency. This was attributed to tiredness $(n=4)$, headache $(n=1)$ or vertigo $(n=1)$, or was not specified $(n=1)$. One person needed to sit down for a while after experiencing instability when working at 
Table 3 Reported symptoms by scanner category*

\begin{tabular}{|c|c|c|c|c|c|c|c|}
\hline \multirow{3}{*}{$\begin{array}{l}\text { Scanner category } \\
\text { Unexposed }\end{array}$} & \multirow{3}{*}{$\begin{array}{l}\text { Overall } \mathbf{n}_{\text {obs }} \\
134\end{array}$} & \multicolumn{6}{|c|}{ Reporting of at least one symptom within the specified category $\left(n_{\text {obs }}(\%)\right)$} \\
\hline & & \multicolumn{2}{|c|}{$\begin{array}{l}\text { SMF target symptoms } \\
(\%)\end{array}$} & \multicolumn{2}{|c|}{$\begin{array}{l}\text { SMF target-core } \\
\text { symptoms (\%) }\end{array}$} & \multicolumn{2}{|c|}{$\begin{array}{l}\text { A priori unrelated } \\
\text { symptoms (\%) }\end{array}$} \\
\hline & & 25 & $(19)$ & 1 & (1) & 4 & (3) \\
\hline 1.5 T closed boret & 259 & 72 & $(28)$ & 14 & (5) & 17 & (7) \\
\hline $3 \mathrm{~T}$ closed bore $\ddagger$ & 131 & 46 & $(35)$ & 12 & (9) & 3 & (2) \\
\hline $7 \mathrm{~T}$ closed bore§ & 31 & 12 & (39) & 9 & (29) & 0 & (0) \\
\hline$<1.5 \mathrm{~T}$ various types $\emptyset$ & 49 & 13 & $(27)$ & 2 & (4) & 7 & (14) \\
\hline$>4.7 \mathrm{~T}$ small bore** & 57 & 9 & $(16)$ & 0 & $(0)$ & 4 & (7) \\
\hline Overall & 619 & 162 & (26) & 35 & (6) & 33 & (5) \\
\hline \multicolumn{8}{|c|}{$\begin{array}{l}\text { * } \text { * Scanner category was missing for } 14 \text { of the } 633 \text { observations, resulting in } 619 \text { valid observations. One shift can satisfy more than one scanner category; th } \\
\text { observations exceeds the total number of } 619 \text { observations. Results are based on an unbalanced study sampling design with repeated measures per subject } \\
\text { tIncludes all large-bore cylindrical systems of } 1.5 \text { Tesla. } \\
\text { flncludes all large-bore cylindrical systems of } 3.0 \text { Tesla. } \\
\text { §Includes all large-bore cylindrical systems of } 7.0 \text { Tesla. } \\
\text { IIncludes extremity scanners, upright scanners and open (ie, transversal field) scanners up to } 1.5 \text { Tesla and large-bore cylindrical systems below } 1.5 \text { Tesla. } \\
\text { ** Includes small-bore cylindrical animal scanners with field strengths between } 4.7 \text { and } 11.7 \text { Tesla. }\end{array}$} \\
\hline
\end{tabular}

a 7 Tesla scanner. Two subjects did not specify how their work had been affected.

\section{DISCUSSION}

In this observational study among 331 employees working at 14 clinical and research MRI facilities, one or more of the listed SMF target symptoms were reported during $16-39 \%$ of the MRI work shifts, depending on the MRI system people worked with. There was an increase in the incidence of reported SMF target symptoms when subjects worked with 1.5 Tesla, 3 Tesla and 7 Tesla closed-bore scanners. We observed a positive exposure-response association between scanner field strength of closed-bore scanners and symptom incidence on the same day of exposure. This trend was stronger for reporting of any of five 'core' target symptoms for which stronger prior evidence of an association with SMF exposure was available from previous studies. $^{8} 915$ Interestingly, when we performed an additional analysis for SMF target symptoms when the five SMF core symptoms were omitted from the SMF target symptom definition, effects of exposure did not emerge for the SMF target symptoms category (results not shown). This suggests that the trends observed for SMF target symptoms were mainly driven by the core symptoms category. Two of the five 'core' target symptoms, vertigo and metallic taste, appeared to be mainly driving the observed exposure-response associations. These symptoms were reported during $6 \%$ and $2 \%$ of MRI (ie, SMF exposed) shifts, respectively, and were absent during non-MRI shifts. This supports an association with SMF exposure. Both symptoms were of an acute and short-lasting (ie, transient) nature, with reported symptom durations of less than $15 \mathrm{~min}$.

The results of this study are in agreement with other studies among people working with MRI scanners in similar ${ }^{10}$ or other $^{89}$ work environments that also found positive associations between acute subjective (transient) symptoms and increasing magnetic field strengths of MRI scanners. It is suggested that perception thresholds may exist for certain symptoms.

Since presence in the static magnetic stray field around an MRI scanner exposes the workers to SMF as well as TvMF, the current study design does not enable us to distinguish which type of exposure causes the symptoms. Several studies report that patients and volunteers experienced more symptoms while being moved into an MRI scanner, as opposed to lying still inside the SMF of the scanner bore. ${ }^{14} 18$ This suggests an effect of the TvMF, which was also supported by data from Glover et al. ${ }^{16}$ However, based on the results of recent experimental studies, some scientists argue that vertigo can potentially be evoked by a static field without a time-varying component. ${ }^{26} 27$ With respect to metallic taste, no clear patterns of association with a specific

Table 4 Adjusted* ORs for scanner category in the 'symptom-reporting' class

\begin{tabular}{|c|c|c|c|c|c|c|}
\hline \multirow[b]{2}{*}{ Scanner category } & \multicolumn{2}{|c|}{ SMF target symptoms } & \multicolumn{2}{|c|}{ SMF target-core symptoms } & \multicolumn{2}{|c|}{ A priori unrelated symptoms } \\
\hline & OR & $95 \% \mathrm{Cl}$ & OR & $95 \% \mathrm{Cl}$ & OR & $95 \% \mathrm{Cl}$ \\
\hline Unexposed (reference) & 1 & & 1 & & 1 & \\
\hline 1.5 T closed boret & 1.88 & $1.07-3.31$ & 2.41 & $0.72-8.13$ & 0.75 & $0.17-3.34$ \\
\hline 3.0 T closed bore & 2.14 & $1.13-4.03$ & 4.92 & $1.21-19.97$ & 0.28 & $0.05-1.62$ \\
\hline 7.0 T closed bore§ & 4.17 & $1.30-13.35$ & 66.82 & $6.39-698.97$ & n.a. & n.a. \\
\hline$<1.5$ T various types $\emptyset$ & 1.47 & $0.59-3.64$ & 0.84 & $0.14-5.19$ & 1.53 & $0.25-9.41$ \\
\hline$>4.7 \mathrm{~T}$ small bore** & 0.72 & $0.27-1.94$ & n.a. & n.a. & 17.70 & $0.04-7379.05$ \\
\hline
\end{tabular}


type of magnetic field exposure have yet been established and the underlying mechanism remains unclear. ${ }^{7} 8141828$ More research is needed to determine which type of magnetic field exposure causes symptoms such as vertigo and metallic taste.

Data analysis revealed that the SMF (core) target symptoms in the current study were clustered and were reported by a subgroup of more 'symptom reporting prone' employees. For the other group, despite comparable exposure conditions, no exposuredependent associations between scanner strength and reporting of SMF (core) target symptoms were observed. This suggests that a possible underlying trait of personal sensitivity to SMF-related symptoms exists and, thus, we postulate that the probability of experiencing SMF-related symptoms is determined by a combination of personal sensitivity and level of exposure to static or TvMFs. A next issue to be addressed with respect to MRI-related symptoms is why some people appear to have a lower threshold (or higher sensitivity) for experiencing symptoms than others. Although some potential modifying factors (eg, age, gender) were already included in our study, additional aspects could be interesting to assess in this context. Future research could focus, for example, on whether history of neurological injury or disease plays a modifying role in the association between SMF or TvMF exposure and symptom incidence-especially injury and diseases related to the vestibular system, since the vestibular system appears to be sensitive to exposure from strong SMFs. ${ }^{27}$ Also, behavioural aspects, such as alcohol consumption patterns and medication use may be interesting to study.

For two scanner categories, symptom incidence did not significantly differ from that for unexposed shifts: Small-bore animal scanners (4.7-11.7 Tesla) and various scanner types up to 1.5 Tesla (including closed-bore scanners with a field strength below 1.5 Tesla, and open scanners, upright scanners and extremity scanners with field strengths up to 1.5 Tesla). This may possibly be attributed to lower SMF and TvMF exposure in these two scanner groups. With the exception of the closed-bore scanners below 1.5 Tesla, the distribution of the static magnetic stray fields around these scanners and the work practice required when working with them differ considerably from the more commonly used large closed-bore scanners. Consequently, exposure patterns around these scanners are not comparable to large closed-bore scanners, and an increase in scanner field strength does not necessarily imply an increase in potential SMF and TvMF exposure as is the case for large closed-bore scanners. Nevertheless, since the 'various scanner types up to 1.5 Tesla' and 'small-bore animal scanners' categories include various types of scanners and field strengths, and not enough data were available to analyse these separately, it cannot be excluded that one or more specific scanner types within these categories may be associated with increased reporting of symptoms.

Despite a moderate reporting of presence inside a scanner room during image acquisition (13\% of the shifts), specific symptoms related to SGF exposure (muscle contractions; tingling sensation) were rarely reported by clinical and research MRI staff, and appeared to be associated only with very specific work practices with presumably a high and prolonged exposure to switched-gradient fields.

To date, this is the largest study to have investigated incidence of MRI-related symptoms in an occupational setting, with data collected from over 300 subjects from various workplaces and exposure conditions, and it is the first to assess how these symptoms affect work practice. The study design included comparison with SMF-unexposed shifts, and facilitated categorisation of exposure based on scanner type and field strength, which allowed for assessment of an exposure-response association. Additionally, potential demographic and workplace-related confounding factors and effect modifiers were taken into account in the statistical analysis, which had not been considered in previous studies among MRI staff or MRI manufacturing employees. ${ }^{6-10}$ The selection of workplaces enabled inclusion of a broad range of scanners, resulting in a large exposure range. However, since the selected MRI facilities were not representative of all MRI facilities in The Netherlands, overall incidence numbers of the total study sample cannot be generalised to the total population of MRI staff in The Netherlands. ${ }^{21}$

We were able to individually analyse subgroups of symptoms. The symptoms in the diary were not specifically assessed in relation to MRI; instead, a range of general and specific symptoms were assessed over the complete work shift, allowing for a more objective assessment of symptoms. A priori unrelated general symptoms were found to be unrelated to exposure to static magnetic stray fields of MRI scanners, indicating employees did not simply overreport symptoms. This serves as additional evidence that SMF target (core) symptoms are very likely related to SMF and/or or motion-induced TvMF exposure. However, in this observational study, a completely objective symptom assessment was not possible, since subjects were aware of their exposure condition (ie, working with MRI or not, and the field strength of the scanner).

Also, the role of personal behaviour should not be ignored; people may adjust their work behaviour to reduce or avoid experiencing symptoms through lowering their pace when walking into the stray field in the direction of the scanner, thus lowering their exposure to TvMF. This is a matter of reverse causality, where the outcome (in this case experiencing acute SMF-related symptoms) may affect the exposure status. Furthermore, MRI staff who are very sensitive to this exposure, to the extent that it heavily affects their work, might avoid working with MRI scanners at all.

Because of the observational nature of this study, job titles were not equally distributed over the exposure conditions. Small-bore animal scanners and 7 Tesla closed-bore scanners, for example, were used mainly by researchers and technical staff, whereas other scanners were predominantly used by clinical staff. The non-MRI shifts in this study (ie, the SMF-unexposed condition) were mainly performed by (MRI and CT) radiographers. Therefore, we additionally examined symptom incidence among a subset of radiographers only, who constituted the largest occupational group in this study. This yielded similar patterns as were observed among all participants (results not shown).

A subanalysis among shifts where people worked with only one scanner showed that the number of scanners one worked with during a shift did not affect symptom-reporting patterns (results not shown). This suggests that the exposure categorisation used for people who worked with more than one scanner did not bias the outcomes.

In conclusion, for those employees who did report symptoms, this study indicated an increased incidence of experiencing specific transient 'MRI-related' symptoms among healthcare and research staff working with closed-bore MRI scanners of 1.5-7 Tesla. Incidence of symptoms was positively associated with scanner magnet strength. This positive association was mainly driven by reporting of vertigo and metallic taste. With the increasing use of (ultra-)high field MRI systems for scanning human subjects, and technological developments enabling the design of whole-body magnets exceeding 7 Tesla, ${ }^{21} 29$ the number of workers experiencing MRI-related symptoms might increase if scanner design and shielding are not altered. 
Acknowledgements Our thanks go to the coordinators of the participating MRI, $\mathrm{CT}$ and research departments for allowing us to undertake research at their departments. We are especially grateful to all employees who voluntarily participated in the study. Special thanks go to Évelyne Cambron-Goulet, who contributed to the data collection and data management of the data collected at experimental animal MRI facilities.

Contributors KS designed the study protocol and data collection tools, organised, monitored and conducted the data collection process, cleaned and analysed the data, drafted and revised the paper, and is guarantor. YC co-designed the study protocol and data collection tools, advised in the data cleaning and analysis process, and drafted and revised the paper. CKM cleaned and analysed data, and revised the draft paper. FdV initiated the project, designed the study outline and revised the draft paper. LP designed and conducted the statistical analyses, and revised the draft paper. HK initiated the project, designed the study outline, co-designed the study protocol and data collection tools, advised in the data cleaning and analysis process, drafted and revised the paper, and is guarantor.

Funding This work was supported by The Netherlands Organisation for Health Research and Development (ZonMw) within the programme Electromagnetic Fields and Health Research, under grant numbers 85100001 and 85800001 . The funding source had no involvement in the study design; in the collection, analysis and interpretation of data; in the writing of the report; or in the decision to submit the paper for publication.

\section{Competing interests None.}

Ethics approval Medical Research Ethics Committee of the Utrecht University Medical Center.

Provenance and peer review Not commissioned; externally peer reviewed.

Open Access This is an Open Access article distributed in accordance with the Creative Commons Attribution Non Commercial (CC BY-NC 3.0) license, which permits others to distribute, remix, adapt, build upon this work non-commercially, and license their derivative works on different terms, provided the original work is properly cited and the use is non-commercial. See: http://creativecommons.org/ licenses/by-nc/3.0/

\section{REFERENCES}

1 Chadwick P. (citing work by Crozier). Assessment of electromagnetic fields around magnetic resonance imaging (MRI) equipment. UK Health and Safety Executive research report. 2007;RR570. http://www.hse.gov.uk/research/rrpdf/rr570.pdf (accessed 11 Feb 2014)

2 Glover PM. Interaction of MRI field gradients with the human body. Phys Med Biol 2009;54:R99-R115.

3 Reilly JP. Principles of nerve and heart excitation by time-varying magnetic fields. Ann N Y Acad Sci 1992;649:96-117.

4 McRobbie DW. Occupational exposure in MRI. Br J Radiol 2012;85:293-312.

5 Shellock FG, Crues JV. MR procedures: Biologic effects, safety, and patient care. Radiology 2004;232:635-52.

6 Capstick M, McRobbie D, Hand J, et al. An investigation into occupational exposure to electromagnetic fields for personnel working with and around medical magnetic resonance imaging equipment. Report on Project VT/2007/017 for the European Commission Employment, Social Affairs and Equal Opportunities DG. 2008. http:// www.myesr.org/html/img/pool/VT2007017FinalReportv04.pdf (accessed 11 Feb 2013).
7 Cavin ID, Glover PM, Bowtell RW, et al. Thresholds for perceiving metallic taste at high magnetic field. J Magn Reson Imaging 2007;26:1357-61.

8 de Vocht $\mathrm{F}$, van Drooge $\mathrm{H}$, Engels $\mathrm{H}$, et al. Exposure, health complaints and cognitive performance among employees of an MRI scanners manufacturing department. J Magn Reson Imaging 2006;23:197-204.

9 Schenck JF, Dumoulin CL, Redington RW, et al. Human exposure to 4.0-tesla magnetic fields in a whole-body scanner. Med Phys 1992;19:1089-98.

10 Wilén J, De Vocht F. Health complaints among nurses working near MRI scanners-a descriptive pilot study. Eur J Radiol 2011;80:510-3.

11 Yang $M$, Christoforidis G, Abduljali A, et al. Vital signs investigation in subjects undergoing MR imaging at 8 T. AJNR Am J Neuroradiol 2006;27:922-8.

12 Versluis MJ, Teeuwisse WM, Kan HE, et al. Subject tolerance of $7 \mathrm{~T} \mathrm{MR}$ examinations. J Magn Reson Imaging 2013;38:722-5.

13 Theysohn JM, Maderwald S, Kraff 0 , et al. Subjective acceptance of 7 Tesla MRI for human imaging. MAGMA 2008;21:63-72.

14 Heilmaier C, Theysohn JM, Maderwald S, et al. A large-scale study on subjective perception of discomfort during 7 and 1.5 T MRI examinations. Bioelectromagnetics 2011;32:610-19.

15 Heinrich A, Szostek A, Meyer P, et al. Cognition and sensation in very high static magnetic fields: a randomized case-crossover study with different field strengths. Radiology 2013;266:236-45.

16 Glover PM, Cavin I, Qian W, et al. Magnetic-field-induced vertigo: A theoretical and experimental investigation. Bioelectromagnetics 2007;28:349-61.

17 Atkinson IC, Renteria L, Burd H, et al. Safety of human MRI at static fields above the FDA 8 T guideline: sodium imaging at $9.4 \mathrm{~T}$ does not affect vital signs or cognitive ability. J Magn Reson Imaging 2007;26:1222-7.

18 Atkinson IC, Sonstegaard R, Pliskin NH, et al. Vital signs and cognitive function are not affected by 23-sodium and 17-oxygen magnetic resonance imaging of the human brain at 9.4 T. J Magn Reson Imaging 2010;32:82-7.

19 van Nierop LE, Slottje P, van Zandvoort MJ, et al. Effects of magnetic stray fields from a 7 Tesla MRI scanner on neurocognition: a double-blind randomised crossover study. Occup Environ Med 2012;69:759-66.

20 Weintraub MI, Khoury A, Cole SP. Biologic effects of 3 Tesla (T) MR imaging comparing traditional 1.5 T and 0.6 T in 1023 consecutive outpatients. J Neuroimaging 2007;17:241-5.

21 Schaap K, Christopher-De Vries Y, Slottje P, et al. Inventory of MRI applications and workers exposed to MRI-related electromagnetic fields in the Netherlands. Eur $J$ Radiol 2013;82:2279-85.

22 Gruen B, Leisch F. FlexMix Version 2: finite mixtures with concomitant variables and varying and constant parameters. J Stat Softw 2008;28:1-35.

23 Gruen B, Leisch F. Fitting finite mixtures of generalized linear regressions in $\mathrm{R}$. Comput Stat Data Anal 2007;51:5247-52.

24 Leisch F. FlexMix: a general framework for finite mixture models and latent class regression in R. J Stat Softw 2004;11:1-18.

25 Akaike H. A new look at the statistical model identification. IEEE Trans Automat Contr 1974;19:716-23.

26 Mian OS, Li Y, Antunes A, et al. On the vertigo due to static magnetic fields. PLOS ONE 2013;8:e78748.

27 Antunes A, Glover PM, Li Y, et al. Magnetic field effects on the vestibular system: calculation of the pressure on the cupula due to ionic current-induced Lorentz force. Phys Med Biol 2012;57:4477-87.

28 Chakeres DW, De Vocht F. Static magnetic field effects on human subjects related to magnetic resonance imaging systems. Prog Biophys Mol Biol 2005;87:255-65.

29 Gowland PA. Present and future magnetic resonance sources of exposure to static fields. Prog Biophys Mol Biol 2005;87;175-83. 